\title{
Functional Improvement Following Revision Surgery in a Patient with the Dual Disability of a Complicated Residual Limb and Contralateral Hemiplegia: A Case Report
}

\author{
Ki Hyun Byun, Dong Seok Yang, Baek Hee Jang \\ Department of Physical Medicine and Rehabilitation, Ulsan University Hospital, University of Ulsan College of Medicine, Ulsan, Korea
}

\begin{abstract}
The number of reported cases with dual disability is increasing for the past few decades. Currently, dual disability of lower limb amputation and motor weakness after stroke became a strong issue in public concern. The functional levels of patients have shown in the wide range from independent community ambulation to non-ambulation. Thus, it indicates that favorable outcomes for dual disability may depend upon adopted rehabilitative strategies. We present the case of a man with left below-knee amputation and severe right-sided weakness following a huge putaminal hemorrhage. He had suffered from extreme pain and misfit of the prosthetic socket and the complicated residual limb for three years prior to the stroke. Forty days post-stroke, we performed a revision surgery to resolve the complications of bony overgrowth, verrucous hyperplasia, and neuroma and applied an ankle foot orthosis (AFO). Two years post-stroke, he was able to ambulate outside his home and negotiate stairs using a cane. This is the first case with the dual disability of lower limb amputation and contralateral hemiplegia to undergo revision surgery. The results suggest that an early revision surgery and use of an AFO are crucial for achieving a higher level of mobility in such cases.
\end{abstract}

Keywords: Disability, Hemiplegia, Amputation, Surgery

\section{INTRODUCTION}

There have been increasingly important issues in the field of rehabilitation for the patient with the dual disability of lower extremity amputation and hemiplegia following a stroke. Recent advances in prosthetic techniques and the enhanced life expectancy have improved the patient's functional quality of life. Only a few cases with the dual disability of contralateral hemiplegia and amputation have been reported so far. ${ }^{1-8}$ Among these, only one study has analyzed the functional level of amputees with contralateral hemiplegia. ${ }^{4}$ However, in this study, the rehabilitation outcomes differed based on the severity and the sample size. Recovery of the functional level in the cases with a dual disability requires consideration of several factors for reduced functional status such as the contralaterality of the impairment, prosthesis abandonment, women and severity of hemiparesis. ${ }^{3}$ In a previous study, 45 patients with dual disability

Received Aug 10, 2018 Revised Sep 28, 2018

Accepted Oct 25, 2018

Corresponding author Baek Hee Jang

E-mail 0733310@uuh.ulsan.kr had a walker gait in 16 patients, two crutch gait in 16 patients, one crutch or one cane gait in 11 patients, walking without a walking aid in two patients. ${ }^{2}$ In addition, a good fit of the prosthetic socket on the residual limb is essential to achieve a high functional level, which indicates that preserving the residual limb without complications, such as a bony overgrowth, verrucous hyperplasia, calluses, inclusion cyst, allergic dermatitis, and neuroma. ${ }^{9}$

Accordingly, a revision surgery to overcome the complications of lower-limb amputation has demonstrated good surgical outcomes with improved socket comfort and mobility status. ${ }^{10}$

To the best of our knowledge, we present the first case of revision surgery in a patient with a complicated residual limb and contralateral hemiplegia after a stroke, leading to functional improvement. We aimed to investigate the rehabilitation and functional recovery after revision surgery in patients with dual disability of contralateral hemiparesis and amputation. 


\section{CASE REPORT}

A 46-year-old man was referred to our department after experiencing a left putaminal hemorrhage 10 days prior to his visit (Figure 1).

On physical examination, with the Medical Research Council, muscle power revealed grade 0 on the right side and grade 5 on the left side. The patient also presented with global aphasia. Past history revealed that the patient underwent a left below-knee amputation due to a car accident at the age of nine years. Until the stroke, he worked as a pizza delivery man but had difficulty negotiating stairs due to the poorly fitted prosthetic socket and extreme pain in the residual lower limb for the last three years.

We observed that the distal part of the residual limb had a fish mouth shape. The X-ray showed a bony overgrowth of tibia and fibula, and computerized tomography revealed a neuroma and muscle atrophy on the distal residual limb. Twenty days after the onset, a motor evoked potential and a diffusion tensor tractography were performed to predict the possibility of motor recovery. The results indicated that the left corticospinal tract was completely damaged, which implied that a recovery of the right ankle muscle power was impossible (Figure 2). We applied an AFO to the left ankle joint at an early stage to reduce spasticity and contracture. Forty days after the onset, a revision surgery was performed to resolve the complications of the residual limb (Figure 3). Revision surgery includes bone cutting for bone extrusion and excisional biopsy for neuroma and papilomatous changes in stump skin. We amputated the distal part

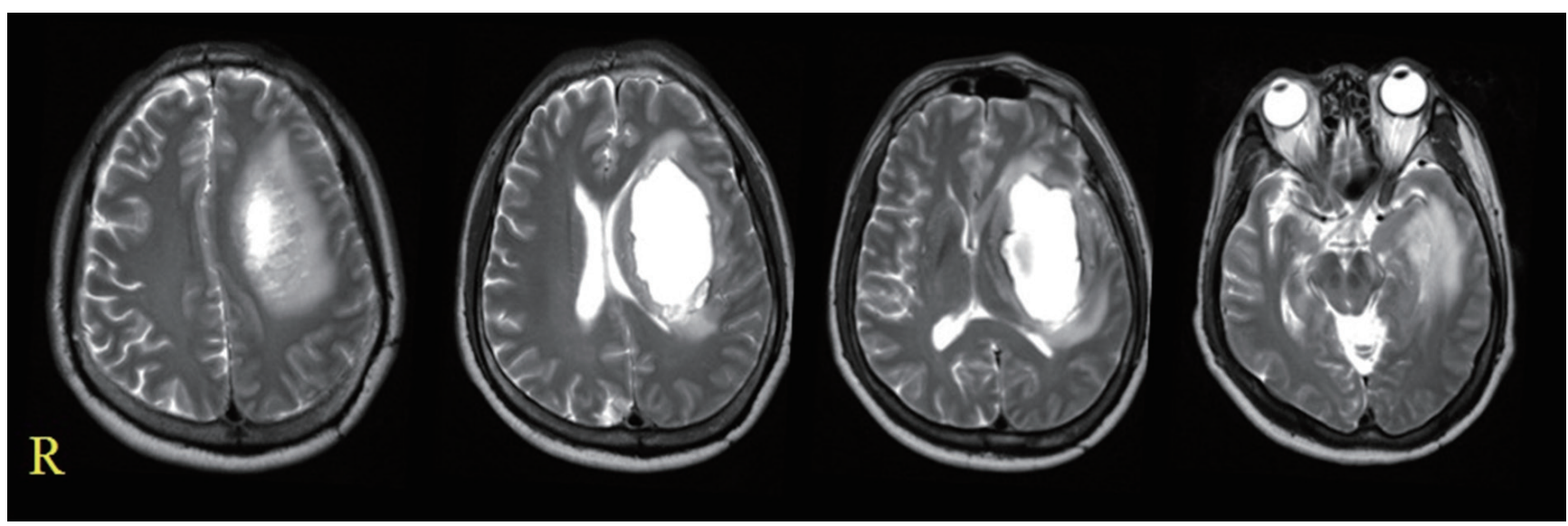

Figure 1. T2 weighted magnetic resonance image revealed a huge hematoma on the left basal ganglia, extending from the cortical area to the temporal area. The huge hematoma on the left hemisphere compressed the right hemisphere, and the midline was shifted to the right hemisphere.

R: right.
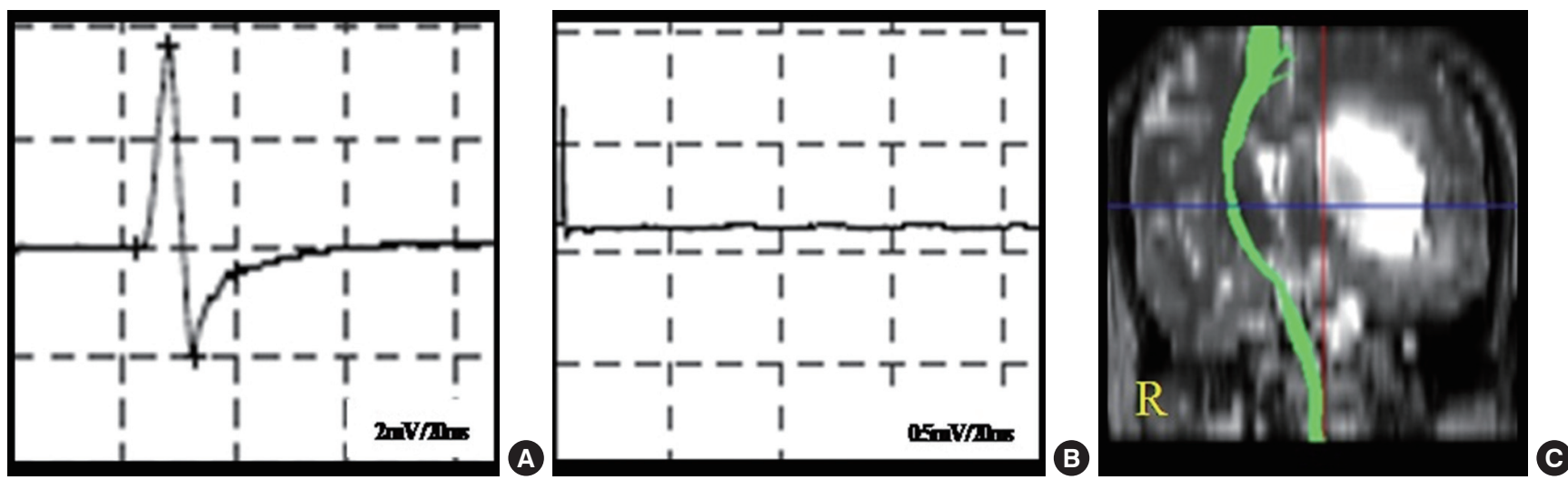

Figure 2. The motor evoked potential obtained from the left abductor pollicis brevis had a latency of $22.8 \mathrm{~ms}$ and an amplitude of $5.650 \mathrm{mV}$ (A). The right abductor pollicis brevis showed no response (B). A diffusion tensor tractography revealed the normal integrity of the right corticospinal tract but not that of the left corticospinal tract (C).

R: right. 

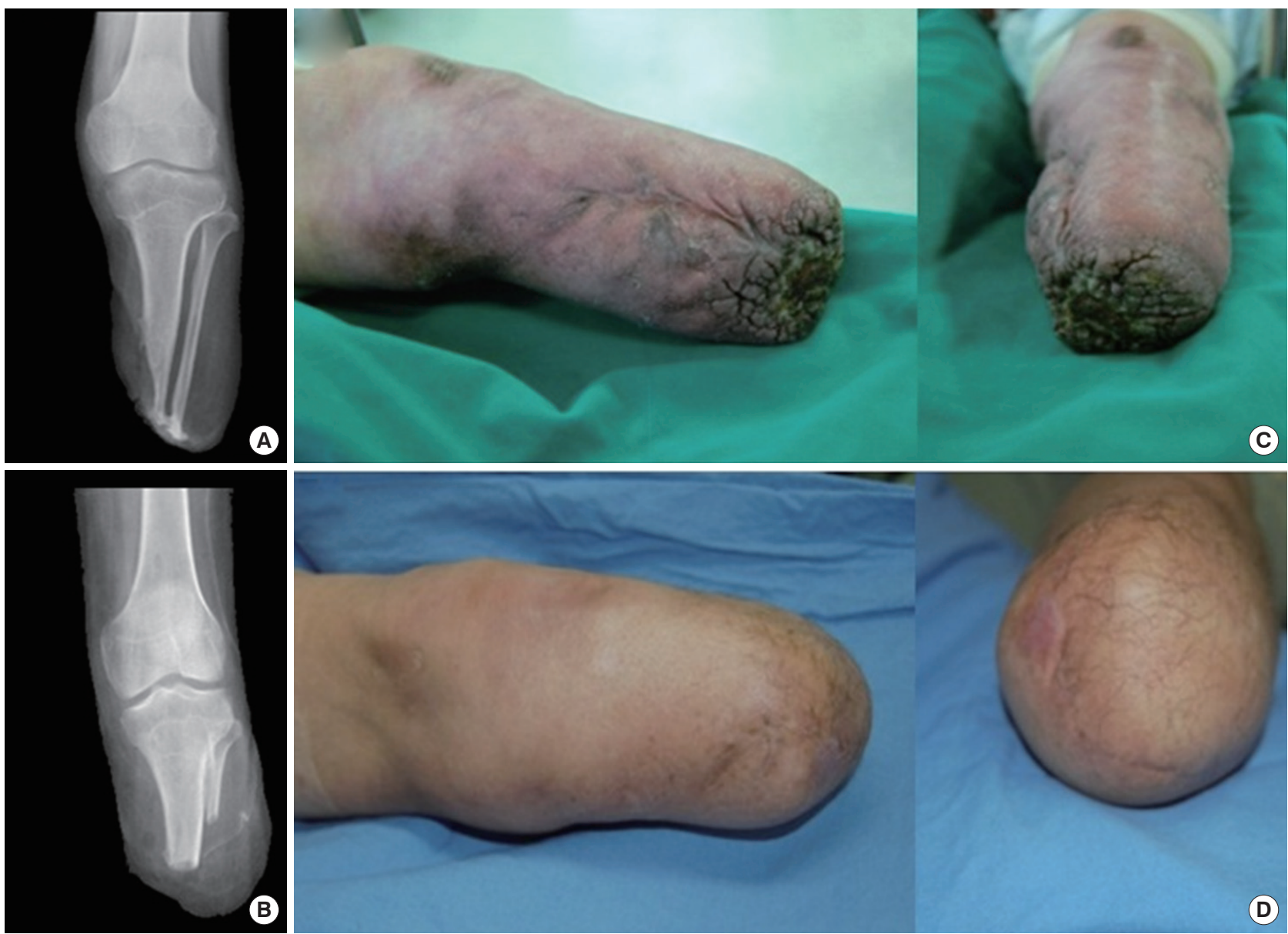

Figure 3. The X-ray showed an overgrowth on the distal part of the tibia and fibula, and the tibial length was $17 \mathrm{~mm}$ shorter than the fibular length (A). Two days after the revision surgery of the complicated residual limb, $10 \mathrm{~cm}$ of the residual limb length from the proximal end of tibia was preserved (B). Before surgery, the stump presented with complications, including verrucous hyperplasia and contact dermatitis. The surface of the residual limb had a fish-mouth shape (C). The site of amputation showed a well-made stump after the revision surgery (D).
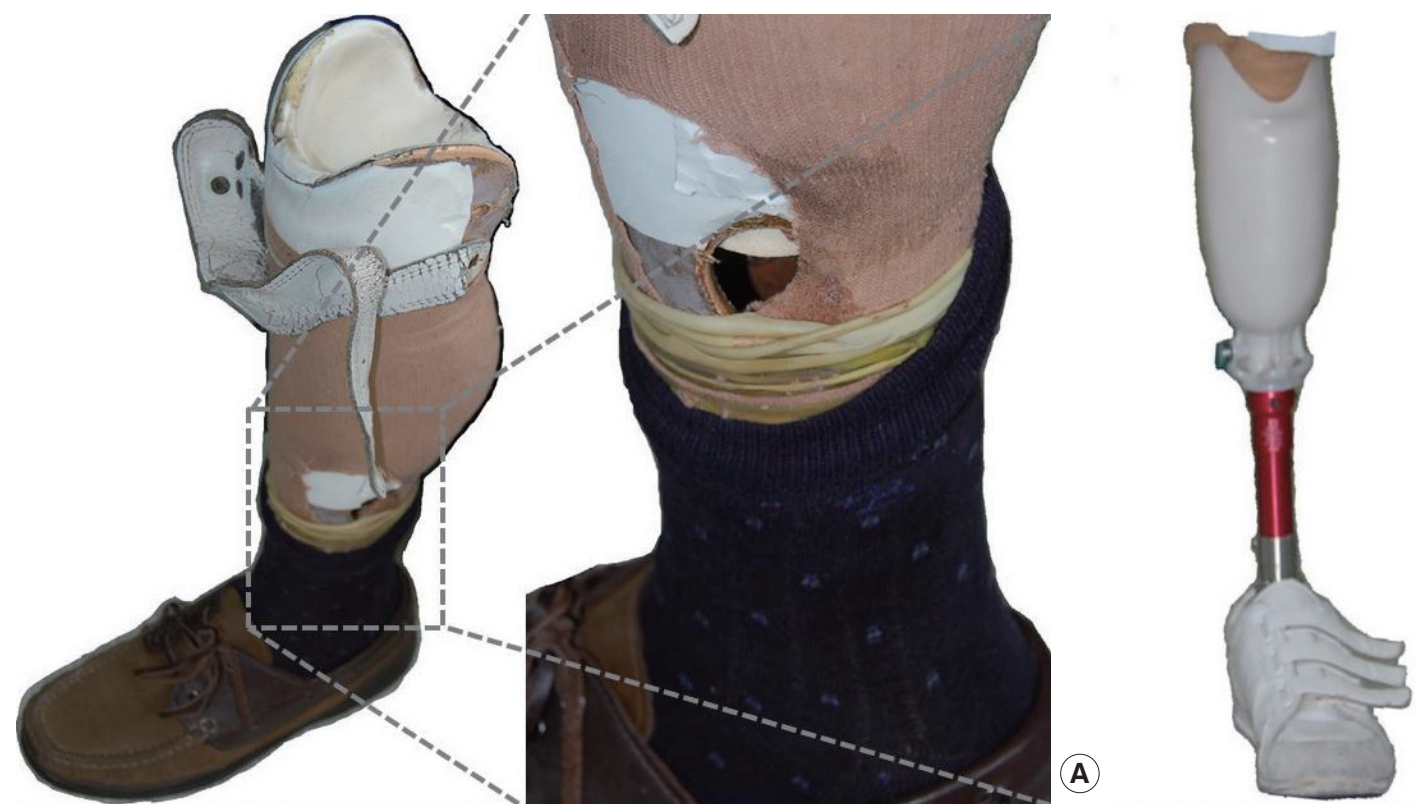

(B)

Figure 4. The prosthesis used in the past and its magnified view (A) and newly produced prosthesis (B). 
$10 \mathrm{~cm}$ from the knee joint, and then performed a myoplasty using the posterior calf muscles. Three months after the stroke, a new prosthesis was manufactured with the silicone suction sockets, patellar tendon weight bearing, supracondylar socket, iron-endoskeletal shank, and single-axis-cushion-heel. During standing training with prosthesis, a $0.5 \mathrm{~cm}$ bullae was formed in the stump distal area. Skin care and weight bearing were prohibited for one week and socket tilting was performed (Figure 4).

The patient participated in a physical therapy session of $40 \mathrm{~min}-$ utes, twice daily, for five days/week. Rehabilitation therapy was performed once daily for occupational therapy and once for physical therapy, including residual limb strengthening about hip extensor, posture and standing balance training, transfer training and donning doffing training.

The physician recorded the Modified Barthel Index score at admission. At the time of admission, 30 points were discharged and 52 points were checked. Eight months after the onset, the patient was able to walk independently for an approximate distance of $20 \mathrm{~m}$ using a cane. The muscle power of the right side showed partial recovery as follows: all upper extremity muscles had a poor muscle power grade with that of the wrist and finger muscles being grade zero, and the muscle power grade of the lower extremity was poor for hip flexors, fair for knee extensors, and zero for the ankle muscles. After 2 years of onset, the patient was able to walk in a flat area of about $100 \mathrm{~m}$ in 10 minutes using a cane. With the help of one person, posture control and stair negotiation were possible.

\section{DISCUSSION}

This study was the first to demonstrate successful rehabilitation outcomes in a patient with revision surgery of the complicated residual limb and application of an AFO to the contralateral hemiplegic ankle joint.

Previously, seven articles reporting cases of dual disability with recovery of the functional level have been published. Studies on mobility have shown a varied outcome, in the range of 5-44\% for achieving community ambulation and 20-55\% for limited/ indoor ambulation. ${ }^{1-6,8}$ Good mental status, mild hemiparesis, transtibial amputation, and laterality have been found to be significantly positive factors for mobility. In addition, the sequence of disability or age $<60$ years seems to have a better effect on ambulation. However, these results have limitations in that there were no set standard parameters, and the studies involved non-homogeneous groups.

In reviewing the previous studies focused on neuromuscular status, which is regarded as one of the most important factors for assessing the functional level in a patient with a dual disability, we found four articles that utilized the Brunnstrom's stage for assessment of the neuromuscular status as follows: Brunnstrom's stage $\mathrm{V}$ or VI indicates that $53.8 \%$ or $57.1 \%$ have community ambulation and $23.3 \%$ have indoor level ambulation, whereas Brunnstrom's stage below III shows that $30 \%$ has indoor level ambulation and 0 or $30.0 \%$ has community ambulation. ${ }^{4,6}$ Brunelli et al. reported that 13 out of 45 cases (28.8\%) with above-knee amputation and hemiparesis (Brunnstrom's stage V or VI) were able to walk with a cane or without any aid. ${ }^{2,3}$ Although we found that all the 12 patients with below-knee amputation could walk independently without any aid, these results do not apply directly to our case as the level of amputation was different and also due to the exclusion of patients with severe weakness.

It is interesting to note that our patient was able to perform community ambulation despite severe weakness. We postulate that the following steps may contribute to good outcomes. First, performing a diffusion tensor tractography at an early time point and measuring a motor evoked potential could predict the integrity of the corticospinal tract. We found that the patient had a complete corticospinal tract injury with no skillful voluntary movement of the finger and ankle. ${ }^{11}$ Second, we recommended an AFO on the hemiplegic side to decrease the risk of falls and ankle contracture. Previously, the patients with contralateral hemiplegia and partial foot amputation have shown improvement in balance and walking distance with an AFO on the hemiplegic side. ${ }^{12,13}$ Third, we performed an early revision surgery on the complicated residual limb 40 days after the stroke. The residual limb had a bony overgrowth, verrucous hyperplasia, and neuroma, and the patient was unable to stand due to extreme pain and a poor fitting of the prosthesis and socket.

Bourke et al. ${ }^{11}$ reported good surgical outcomes and improvement in socket comfort and mobility in 71 cases of revision surgery on lower limb amputation. The rates of surgical success for bony pathology, soft tissue, and pain were $84.6 \%, 81.8 \%$, and $50.0 \%$, respectively. Improvement of socket comfort and mobility were 100\% and $80.8 \%$ in bony pathology, $70.6 \%$ and $47.4 \%$ in soft tissue, and $66.7 \%$ and $12.5 \%$ in pain, respectively. Thus, a revision surgery 
seems to be a promising solution for simultaneously existing complications of bony pathology, soft tissue, and pain.

\section{CONCLUSION}

This is the first reported case of a revision surgery that improved the functional level in regard to community ambulation and ability to negotiate stairs in a patient with the dual disability of contralateral hemiplegia and lower extremity amputation. To achieve favorable outcomes, we suggest as follows. First, prediction of the functional level using electrophysiological study or diffusion tensor tractography is helpful to set up a strategic rehabilitation. Second, a revision surgery to overcome the complications of the residual limb and an AFO for the hemiplegic ankle may be essential.

\section{REFERENCES}

1. Altner PC, Rockley P, Kirby K. Hemiplegia and lower extremity amputation: double disability. Arch Phys Med Rehabil. 1987;68(6):378-9.

2. Brunelli S, Averna T, Porcacchia P et al. Functional status and factors influencing the rehabilitation outcome of people affected by above-knee amputation and hemiparesis. Arch Phys Med Rehabil. 2006;87(7):9951000.

3. Brunelli S, Fusco A, Iosa M et al. Mid- to long-term factors influencing functional status of people affected by lower-limb amputation associated with hemiparesis due to stroke. Disabil Rehabil. 2013;35(12):982-9.

4. Chiu CC, Chen CE, Wang TG et al. Influencing factors and ambulation outcome in patients with dual disabilities of hemiplegia and amputation. Arch Phys Med Rehabil. 2000;81(1):14-7.

5. Kullmann L, Endres M. Rehabilitation of hemiplegic amputees. Int J Rehabil Res. 1987;10(2):159-65.

6. PG OC, Gnatz S. Hemiplegia and amputation: rehabilitation in the dual disability. Arch Phys Med Rehabil. 1989;70(6):451-4.

7. Smith HG, Lerwick ER. Left above-the-knee amputation and right hemiplegia. Phys Ther Rev. 1958;38(10):687-8.

8. Varghese G, Hinterbuchner C, Mondall P et al. Rehabilitation outcome of patients with dual disability of hemiplegia and amputation. Arch Phys Med Rehabil. 1978;59(3):121-3.

9. Dudek NL, Marks MB, Marshall SC. Skin problems in an amputee clinic. Am J Phys Med Rehabil. 2006;85(5):424-9.

10. Bourke HE, Yelden KC, Robinson KP et al. Is revision surgery following lower-limb amputation a worthwhile procedure? A retrospective review of 71 cases. Injury. 2011;42(7):660-6.

11. Cho HM, Choi BY, Chang CH et al. The clinical characteristics of motor function in chronic hemiparetic stroke patients with complete corticospinal tract injury. NeuroRehabilitation. 2012;31(2):207-13.

12. Cakar E, Durmus O, Tekin L et al. The ankle-foot orthosis improves balance and reduces fall risk of chronic spastic hemiparetic patients. Eur J Phys Rehabil Med. 2010;46(3):363-8.

13. Ishii M, Yamanaka T. Leg orthosis treatment for a patient with left parietal foot amputation and right hemiplegia. Top Stroke Rehabil. 2004; 11(3):16-8. 